\title{
BIASED INITIAL DISTRIBUTION FOR SIMULA- TION OF QUEUES WITH A SUPERPOSITION OF PERIODIC AND BURSTY SOURCES
}

\author{
Fumio Ishizaki \\ The University of Tokushima, 2-1 Minamijosanjima, Tokushima 770-8506, Japan \\ ishizaki@is.tokushima-u.ac.jp
}

\begin{abstract}
In this paper, we focus on queues with a superposition of periodic and bursty sources where the initial states of the sources are randomly and independently selected. The dynamics of the queue are described as a reducible Markov chain. For such queues, we consider the tail probability of the queue length as the performance measure and try to estimate it by simulations. To do simulation efficiently, we develop a sampling technique which replaces the initial distribution of the reducible Markov chain with a new biased one. Simulation results show that the sampling technique is useful to reduce the variance of the estimates.
\end{abstract}

Keywords: Importance Sampling, Monte Carlo Simulation, Queues

\section{INTRODUCTION}

In ATM networks, the arrival process of cells is essentially a superposition of sources which are typically bursty and periodic either due to their origin or their periodic slot occupation after traffic shaping. For instance, VBR (variable bit rate) MPEG traffic, which will become a large volume of traffic in future high-speed networks, has both bursty and periodic natures because of its coding scheme. Thus, queues with a superposition of periodic and bursty sources have been studied by many researchers.

Queues with a superposition of periodic sources have the following special characteristic: The dynamics of the queues are not ergodic due to the periodicity of the sources, when the periodic sources are randomly superposed (i.e., the initial states of the sources are randomly chosen). As a result, depending on the combination of the initial states, the system performance such as cell loss ratio and queueing delay can drastically change [9]. This situation will be observed in Section 5.

Unfortunately, it is difficult to estimate the performance in such queues. For example, since the matrix analytical approaches [12] require to deal with prohibitively large size matrices, the analysis is extremely complicated. On the other hand, by standard Monte Carlo (MC) simulations, it is hard to get reliable 
estimates of the performance measure. The reason is that there are rare events whose contribution to the performance measure cannot be neglected. In other words, there exist some bad initial state combinations which lead to extreme degradation of the system performance, although they are rarely realized.

In this paper, we focus on queues with a superposition of periodic and bursty sources where the initial states of the sources are randomly and independently selected. The dynamics of the queue are described as a reducible bivariate Markov chain (i.e., they are not ergodic). We consider the tail probability of the queue length as the system performance, and try to estimate it by simulations. In order to overcome the difficulty in MC simulations, we develop a sampling technique which is a kind of importance sampling (IS) technique (see, e.g., $[3 ; 6 ; 13 ; 16]$ and references therein). In most of the previous works (including IS based on large deviation theory [14], RESTART [15], DPR [7] and so on), ergodic queues have been considered, and replacing only the state transition probabilities with new biased ones has been investigated. Contrary to the previous works, the sampling technique developed in this paper replaces the initial distribution with a new biased one. This means that the realization probabilities of the combinations of the initial states are modified. Existing IS techniques and our sampling technique are not exclusive but complementary. We can expect that combined with existing IS techniques, our sampling technique will lead to more efficient MC simulations.

In order to get an appropriate biased initial distribution, we apply the asymptotic queueing analysis (see, e.g., $[1 ; 2 ; 4 ; 5]$ and references therein). Unfortunately, for this purpose, most of the asymptotic queueing analyses are not suitable. The reason is that they do not take the effect of the initial state combinations on the system performance into account. In contrast, taking the initial state combinations into account, Ishizaki and Takine [8] have studied discretetime queues where the arrival process is a superposition of the general periodic Markov sources. They have derived bound formulas for the asymptotic tail distribution of the queue length. We consider a sample path version of their upper bound as a rough estimate of the queue length and use it to get a biased initial distribution.

The remainder of the paper is organized as follows. In Section 2, we describe a queue with a superposition of periodic and bursty sources. The background material for the asymptotic queueing analysis [8] is provided in Section 3. In Section 4, we develop the sampling technique which replaces the initial distribution with a new biased one. In Section 5, we provide simulation results to examine the usefulness of the sampling technique. Conclusions are drawn in Section 6.

\section{MODEL}

We consider a discrete-time infinite-buffer queueing system. The arrival process at the system is a superposition of $K$ general periodic Markov sources [ 8]. The $k$ th $(k=1, \ldots, K)$ source is governed by an underlying discrete-time $M^{(k)}$-state Markov chain with period $R^{(k)}$. We assume that the number of cells from each source arriving in a slot depends on the state of the underlying 
Markov chain in the slot. The service time of cell is assumed to be constant and equal to one slot.

We now describe our model in more detail. We first introduce notations for each source. Let $S_{n}^{(k)}(n=0,1, \ldots)$ denote a random variable representing the state of the underlying Markov chain of the $k$ th source in the $n$th slot, where $S_{n}^{(k)} \in \mathcal{S}^{(k)}=\left\{0,1, \ldots, M^{(k)}-1\right\}$. We assume that all the underlying Markov chains are irreducible and stationary. The state transition matrix of the underlying Markov chain is denoted by $\boldsymbol{U}^{(k)}=\left\{U_{i, j}^{(k)}\right\}\left(i, j=0, \ldots, M^{(k)}\right.$ 1). We denote the stationary state vector of the underlying Markov chain for the $k$ th source by $\pi^{(k)}=\left(\pi_{0}^{(k)}, \ldots, \pi_{M^{(k)}-1}^{(k)}\right)$. Let $D_{r}^{(k)}$ denote the $r$ th moving class of the $k$ th source, i.e., if $i \in D_{r}^{(k)}\left(r=0, \ldots, R^{(k)}-1\right)$, then $\operatorname{Pr}\left\{S_{n+1}^{(k)}=j \mid S_{n}^{(k)}=i\right\}=0$ for all $j \notin D_{r \oplus_{k} 1}^{(k)}$, where $\oplus_{k}$ is defined as $r \oplus_{k} l=(r+l) \bmod R^{(k)}$ for $k=1, \ldots, K$. Let $P^{(k)}(k=1, \ldots, K)$ denote a random variable representing the index of the moving class where the $k$ th source is in the 0th slot. We then have $S_{0}^{(k)} \in D_{P^{(k)}}^{(k)}$. Hereafter, we call the random variable $P^{(k)}$ the phase of the $k$ th source.

Let $A_{n}^{(k)}(n=1,2, \ldots)$ denote a random variable representing the number of cells arriving from the $k$ th source in the $n$th slot. $\left\{A_{n}^{(k)}\right\}$ is governed by the underlying Markov chain for the $k$ th source. We assume that given $S_{n}^{(k)}$, $A_{n}^{(k)}$ is conditionally independent of all other random variables. Let $\hat{a}_{i j}^{(k)}(l)$ denote the conditional joint probability of the following events: $l$ cells arrive from the $k$ th source and the underlying Markov chain is in state $j$ in the next slot given that the underlying Markov chain is in state $i$ in the current slot. Namely, $\hat{a}_{i j}^{(k)}(l)=\operatorname{Pr}\left\{A_{n+1}^{(k)}=l, S_{n+1}^{(k)}=j \mid S_{n}^{(k)}=i\right\}$. Let $\boldsymbol{A}^{(k)}(l)$ denote an $M^{(k)} \times M^{(k)}$ matrix whose $(i, j)$ th element is given by $\hat{a}_{i j}^{(k)}(l)$. Note that $\boldsymbol{A}^{(k)}(l)$ represents the transition matrix of the underlying Markov chain when $l$ cells arrive from the $k$ th source at the system. We define the probability matrix generating function for the arrival process: $\hat{\boldsymbol{A}}^{(k)}(z)=\sum_{l=0}^{\infty} \boldsymbol{A}^{(k)}(l) z^{l}$. Let $\rho^{(k)}$ denote the traffic intensity of the $k$ th source which is given by $\rho^{(k)}=$ $\boldsymbol{\pi}^{(k)} \sum_{l=0}^{\infty} l \boldsymbol{A}^{(k)}(l) \boldsymbol{e}$, where $\boldsymbol{e}$ is an $M^{(k)} \times 1$ vector whose elements are all equal to one.

Next we consider a superposition of the $K$ general periodic Markov sources. We assume that the $K$ periodic sources are independently superposed. This assumption implies that all the phases of sources are independent with each other. Note here that the assumption makes the underlying Markov chain for the superposed process reducible, because of the periodicity of the sources [8]. As a result, the arrival process is not ergodic.

We define a random variable $S_{n} \in \mathcal{S}=\{0, \ldots, M-1\}(n=0,1, \ldots)$ as $S_{n}=f\left(S_{n}^{(1)}, \cdots, S_{n}^{(K)}\right)$, where $M=\prod_{k=1}^{K} M^{(k)}$ and the function $f$ is defined as $f\left(j^{(1)}, \cdots, j^{(K)}\right)=\sum_{i=1}^{K} j^{(i)} \prod_{k=i+1}^{K} M^{(k)} . S_{n}$ then represents 
the state of the underlying Markov chain for the superposed arrival process in the $n$th slot. Hereafter, we use the expressions $S_{n}=\left(S_{n}^{(1)}, \ldots, S_{n}^{(K)}\right)$ and $S_{n}$ interchangeably to express the state of the underlying Markov chain for the superposed arrival process. Let $\boldsymbol{P}$ denote a random vector representing the phase vector of the superposed arrival process, which is defined as $\boldsymbol{P}=$ $\left(P^{(1)}, \ldots, P^{(K)}\right)$.

Let $A_{n}$ denote a random variable representing the number of cells arriving in the $n$th slot. We then have $A_{n}=\sum_{k=1}^{K} A_{n}^{(k)}$. Note that $\left\{A_{n}\right\}$ is not ergodic, though it is stationary. The overall traffic intensity for the superposed arrival process is then given by $\rho=\sum_{k=1}^{K} \rho^{(k)}$. Hereafter we assume that $\rho<1$. Let $X_{n}(n=0,1, \ldots)$ denote a random variable representing the queue length at the beginning of the $n$th slot. Its evolution is described by $X_{n+1}=\left(X_{n}-1\right)^{+}+A_{n+1}$ with $X_{0}=0$, where $(x)^{+}=\max (x, 0)$.

Note that $\left\{\left(X_{n}, S_{n}\right)\right\}(n=0,1, \ldots)$ becomes a reducible bivariate Markov chain. The one-step state transition matrix $\boldsymbol{Q}$ of the Markov chain is given by

$$
\boldsymbol{Q}=\left[\begin{array}{ccccc}
\boldsymbol{A}_{0} & \boldsymbol{A}_{1} & \boldsymbol{A}_{2} & \boldsymbol{A}_{3} & \cdots \\
\boldsymbol{A}_{0} & \boldsymbol{A}_{1} & \boldsymbol{A}_{2} & \boldsymbol{A}_{3} & \cdots \\
\boldsymbol{O} & \boldsymbol{A}_{0} & \boldsymbol{A}_{1} & \boldsymbol{A}_{2} & \cdots \\
\boldsymbol{O} & \boldsymbol{O} & \boldsymbol{A}_{0} & \boldsymbol{A}_{1} & \cdots \\
\vdots & \vdots & \vdots & \vdots & \ddots
\end{array}\right]
$$

where $\boldsymbol{O}$ denotes the $M \times M$ zero matrix and $\boldsymbol{A}_{l}$ is given by

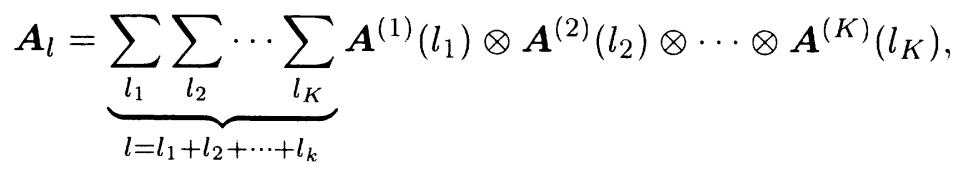

$\otimes$ denotes the Kronecker product. The assumption that all the sources are independent with each other uniquely determines the initial distribution of the reducible bivariate Markov chain. We will provide the form of the initial distribution. To do so, we let $j=f\left(j^{(1)}, \ldots, j^{(K)}\right)$ and $j^{(k)} \in D_{r^{(k)}}^{(k)}(k=$ $1, \ldots, K)$. Then, the initial distribution $\mu$ of the reducible bivariate Markov chain is given by

$$
\mu\left(X_{0}=i, S_{0}=j\right)= \begin{cases}\frac{\prod_{k=1}^{K} q_{j(k), r}^{(k)}(k)}{\prod_{k=1}^{K} R^{(k)}} & (i=0, j=0, \ldots, M-1), \\ 0 & \text { (otherwise) },\end{cases}
$$

where $q_{j, r}^{(k)}$ is defined as

$$
q_{j, r}^{(k)}=\operatorname{Pr}\left\{S_{n}^{(k)}=j \mid S_{n}^{(k)} \in D_{r}^{(k)}\right\} .
$$

We define $g_{N_{0}, N}^{m}$ as $g_{N_{0}, N}^{m}(\omega)=\sum_{n=N_{0}}^{N_{0}+N-1} \mathbf{1}_{\left\{X_{n}>m\right\}}(\omega) / N$, where $\omega$ denotes a sample, $m, N_{0}$ and $N$ are nonnegative integer-valued parameters. 
In this paper, we consider the performance measure $J_{N_{0}, N}^{m}=E\left[g_{N_{0}, N}^{m}\right]=$ $\sum_{n=N_{0}}^{N_{0}+N-1} \operatorname{Pr}\left\{X_{n}>m\right\} / N$.

\section{ASYMPTOTIC QUEUEING ANALYSIS}

In this section, we present the result of the asymptotic queueing analysis as a background material.

Let $\hat{\delta}^{(k)}(z), \hat{\boldsymbol{u}}^{(k)}(z)$ and $\hat{\boldsymbol{v}}^{(k)}(z)$ denote the Perron-Frobenius (PF) eigenvalue for the $k$ th source associated with $\hat{\boldsymbol{A}}^{(k)}(z)$, its associated left and right eigenvectors with normalizing conditions $\hat{\boldsymbol{u}}^{(k)}(z) \hat{\boldsymbol{v}}^{(k)}(z)=\hat{\boldsymbol{u}}^{(k)}(z) \boldsymbol{e}=1$, respectively. The probability matrix generating function $\boldsymbol{A}(z)$ for the superposed arrival process is then given by $\boldsymbol{A}(z)=\hat{\boldsymbol{A}}^{(1)}(z) \otimes \cdots \otimes \hat{\boldsymbol{A}}^{(K)}(z)$. The PF eigenvalue $\delta(z)$ for the superposed arrival process is then given by $\delta(z)=$ $\prod_{k=1}^{K} \hat{\delta}^{(k)}(z)$, and its associated left and right eigenvectors $\boldsymbol{u}(z)$ and $\boldsymbol{v}(z)$ are given by $\boldsymbol{u}(z)=\hat{\boldsymbol{u}}^{(1)}(z) \otimes \cdots \otimes \hat{\boldsymbol{u}}^{(K)}(z)$ and $\boldsymbol{v}(z)=\hat{\boldsymbol{v}}^{(1)}(z) \otimes \cdots \otimes \hat{\boldsymbol{v}}^{(K)}(z)$.

Note that we can easily construct an arrival process in such a way that the queue length is bounded and has no tail. The following assumption guarantees that the queue length (including a cell in service) has a simple asymptotic form [ 8].

\section{Assumption 1:}

- There exists at least one zero of $\operatorname{det}[z \boldsymbol{I}-\boldsymbol{A}(z)]$ outside the unit disk.

- Among those, there exists a real and positive zero $z^{*}$, and the absolute values of $z^{*}$ is strictly smaller than those of other zeros.

- $\boldsymbol{O}<\boldsymbol{A}(z) \ll+\infty, 1 \leq z \leq z^{*}, z \in \mathcal{R}$, where $\mathcal{R}$ denotes a set of all real numbers.

For $\boldsymbol{r}=\left(r^{(1)}, \ldots, r^{(K)}\right)\left(r^{(k)} \in\left\{0, \ldots, R^{(k)}-1\right\}\right.$ for $\left.k=1, \ldots, K\right)$, we define $c(\boldsymbol{r}, z)$ as

$$
c(\boldsymbol{r}, z)=\frac{1}{R} \sum_{l=0}^{R-1} \prod_{k=1}^{K} \theta_{r^{(k)} \oplus_{k} l}^{(k)}(z),
$$

where

$$
\begin{gathered}
\theta_{r}^{(k)}(z)=\sum_{j \in D_{r}^{(k)}} q_{j, r}^{(k)} s_{j}^{(k)} \hat{v}_{j}^{(k)}(z), \\
s_{j}^{(k)}=\operatorname{Pr}\left(A_{n}^{(k)}=0 \mid S_{n}^{(k)}=j\right), \quad \hat{v}_{j}^{(k)}(z)=\left[\hat{\boldsymbol{v}}^{(k)}(z)\right]_{j},
\end{gathered}
$$

$q_{j, r}^{(k)}$ is defined as (1), $R$ is defined as $R=\operatorname{LCM}\left\{R^{(1)}, \ldots, R^{(K)}\right\}$, and $\hat{\boldsymbol{v}}^{(k)}(z)$ denotes the right eigenvectors which is associated with the PF eigenvalue $\hat{\delta}^{(k)}(z)$ and satisfies the normalizing condition. 
The following proposition holds under Assumption 1. From the ergodic theorem [11] and Theorem 4.1 in [8], it immediately follows. The proof is shown in [10].

Proposition 1: Under Assumption 1, for a sufficiently large $m$, any nonnegative integer $N_{0}$ and any phase vector $\boldsymbol{P}$, we have

$$
\lim _{N \rightarrow \infty} \frac{1}{N} \sum_{n=N_{0}}^{N_{0}+N-1} \mathbf{1}_{\left\{X_{n}>m\right\}} \leq \frac{\left(z^{*}\right)^{-m}}{\delta^{\prime}\left(z^{*}\right)-1} c\left(\boldsymbol{P}, z^{*}\right) \quad \text { a.s. }
$$

where $\delta(z)$ denotes the PF eigenvalue for the superposed arrival process and $z^{*}$ is the minimum real solution of

$$
z=\delta(z)
$$

for $z \in(1, \infty)$.

\section{SAMPLING TECHNIQUE}

We consider estimating the performance measure $J_{N_{0}, N}^{m}$ by simulation. In order to do simulation efficiently, we replace the initial distribution $\mu$ of the bivariate Markov chain with a new biased one $\nu$. For this purpose, we use Proposition 1 in the previous section. Recall here that the system is described as a reducible Markov chain.

We let $j^{(k)} \in D_{r^{(k)}}^{(k)}(k=1, \ldots, K), j=f\left(j^{(1)}, \ldots, j^{(K)}\right)$ and $r=$ $\left(r^{(1)}, \ldots, r^{(K)}\right)$. Also, let $\bar{c}(z)$ denote the sum of $c(\cdot, z)$ over all the phase combinations which is defined as

$$
\bar{c}(z)=\sum_{r^{(1)}=0}^{R^{(1)}-1} \cdots \sum_{r^{(K)}=0}^{R^{(K)}-1} c(\boldsymbol{r}, z),
$$

where $c(\cdot, \cdot)$ is defined in (2). We then define the biased initial distribution $\nu$ of the reducible bivariate Markov chain as

$\nu\left(X_{0}=i, S_{0}=j\right)= \begin{cases}\frac{c\left(\boldsymbol{r}, z^{*}\right)}{\bar{c}\left(z^{*}\right)} \prod_{k=1}^{K} q_{j^{(k)}, r^{(k)}}^{(k)} & (i=0, j=0, \ldots, M-1), \\ 0 & \text { (otherwise) },\end{cases}$

where $z^{*}$ is the minimal real solution of $z=\delta(z)$ for $z \in(1, \infty)$.

Roughly speaking, the heuristic idea to get the biased initial distribution $\nu$ is as follows. The optimal biased initial distribution $\nu^{*}$ satisfies [13]

$$
\nu^{*}\left(\mathcal{B}_{0}\right) \operatorname{Pr}\left(\mathcal{B} \mid \mathcal{B}_{0}\right)=\frac{g_{N_{0}, N}^{m}(\omega)}{J_{N_{0}, N}^{m}} \mu\left(\mathcal{B}_{0}\right) \operatorname{Pr}\left(\mathcal{B} \mid \mathcal{B}_{0}\right),
$$

where $\omega$ is any sample satisfying $\omega \in \mathcal{B} \cap \mathcal{B}_{0}, \mathcal{B}$ and $\mathcal{B}_{0}$ are events given by $\mathcal{B}=\left\{X_{1}=i_{1}, S_{1}=j_{1}, \ldots, X_{N_{0}+N-1}=i_{N_{0}+N-1}, S_{N_{0}+N-1}=j_{N_{0}+N-1}\right\}$, 


$$
\mathcal{B}_{0}=\left\{X_{0}=i, S_{0}=j\right\},
$$

respectively. We consider the upper bound given in Proposition 1 as the approximation. If for any $\omega \in \mathcal{B} \cap \mathcal{B}_{0}$ the approximation

$$
g_{N_{0}, N}^{m}(\omega) \approx \frac{\left(z^{*}\right)^{-m}}{\delta^{\prime}\left(z^{*}\right)-1} c\left(\boldsymbol{P}(\omega), z^{*}\right)
$$

is a rough estimate of $g_{N_{0}, N}^{m}$, then $\nu^{*}\left(\mathcal{B}_{0}\right)$ may be expressed as $\nu^{*}\left(\mathcal{B}_{0}\right) \approx$ $A^{\prime} c\left(\boldsymbol{r}, z^{*}\right)$, where $A^{\prime}$ is an unknown constant. Noting that $\boldsymbol{P}(\omega)=\boldsymbol{r}$ for $\omega \in \mathcal{B}_{0}$, we can determine $A^{\prime}$ from the normalizing condition for $\nu$ and get (5).

We now describe MC simulation procedure with the sampling technique which uses the biased initial distribution $\nu$ below.

1. Numerically solve (3) to get $z^{*}$ and calculate $\bar{c}\left(z^{*}\right)$.

2. Do the following steps (a)-(d) for $n=0, \ldots, N_{s}-1$ to get $N_{s}$ samples.

(a) Randomly choose a vector $r=\left(r^{(1)}, \ldots, r^{(K)}\right)$ with probability $c\left(\boldsymbol{r}, z^{*}\right) / \bar{c}\left(z^{*}\right)\left(r^{(k)} \in\left\{0, \ldots, R^{(k)}-1\right\}\right.$ for $\left.k=1, \ldots, K\right)$.

(b) Then, for $k=1, \ldots, K$, randomly select a initial state $j^{(k)}$ for the $k$ th source with probability $q_{j^{(k)}, r^{(k)}}^{(k)}$.

(c) Do simulation with this selected initial state and $X_{0}=0$, and then get the $n$th sample performance $\hat{Y}_{n}$.

(d) Get the unbiased estimator $\hat{Z}_{n}$ by multiplying $\hat{Y}_{n}$ by its likelihood ratio: $\hat{Z}_{n}=\bar{c}\left(z^{*}\right) /\left(c\left(\boldsymbol{r}, z^{*}\right) \prod_{k=1}^{K} R^{(k)}\right) \hat{Y}_{n}$.

3. Finally, obtain an estimate as the sample mean $\bar{Z}$ of the unbiased estimators $\hat{Z}_{n}\left(n=0, \ldots, N_{s}-1\right)$ by $\bar{Z}=\sum_{n=0}^{N_{s}-1} \hat{Z}_{n} / N_{s}$.

Note here that if we set $c\left(\boldsymbol{r}, z^{*}\right)$ constant, the MC simulation procedure with the sampling technique reduces to the standard MC simulation procedure.

When we get the biased initial distribution $\nu$, we need to calculate $\bar{c}\left(z^{*}\right)$. However, for this purpose, we do not need to compute $c\left(\boldsymbol{r}, z^{*}\right)$ for all $\boldsymbol{r}$. Instead, we can compute $\bar{c}\left(z^{*}\right)$ from the following equation:

$$
\bar{c}\left(z^{*}\right)=\prod_{k=1}^{K} \sum_{l=0}^{R^{(k)}-1} \theta_{l}^{(k)}\left(z^{*}\right) .
$$

Recall here that the number of the phase combinations is prohibitively large. Thus, getting $\bar{c}\left(z^{*}\right)$ from (4) is expensive in terms of computational cost. Using (6), we can greatly reduce the computational cost to get $\bar{c}\left(z^{*}\right)$.

In Step 2 (a), we need to select a vector $\boldsymbol{r}$ with the probability $c\left(\boldsymbol{r}, z^{*}\right) / \bar{c}\left(z^{*}\right)$. Since the number of phase combinations is very large, it is not so easy to determine the selected phase combination in a short time. To overcome this difficulty, we use a sophisticated algorithm described as follows: 


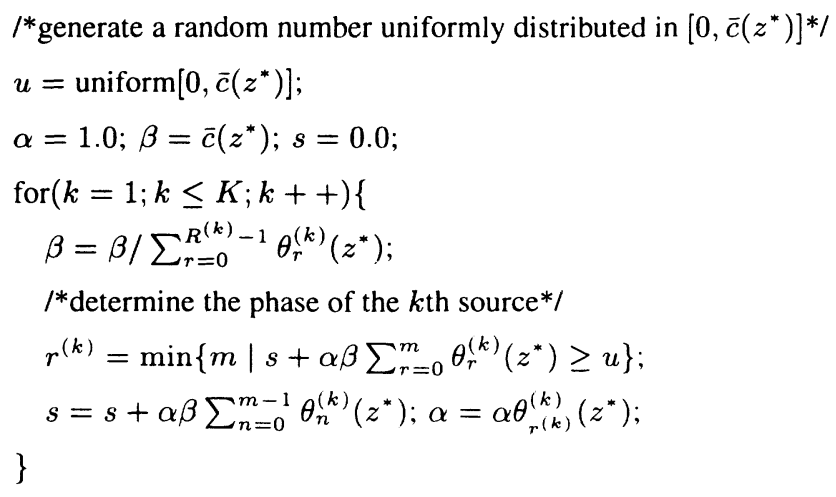

\section{SIMULATION RESULTS}

In this section, we apply the sampling technique to queueing systems where the arrival process is a superposition of periodic Bernoulli sources [9], and examine the usefulness of the sampling technique. For this purpose, we provide some simulation results and compare the variance of the estimates obtained through the MC simulation using the sampling technique with that obtained through the standard MC simulation. Due to limit of space, we show only one simulation example in this paper. Various simulation examples are shown in [ 10].

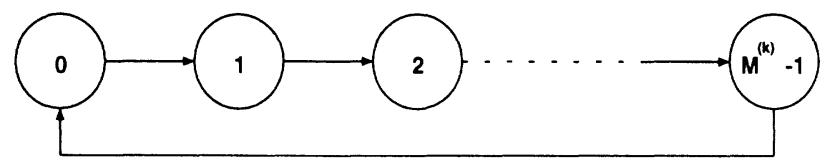

Figure 1 Periodic Bernoulli source

We first describe a periodic Bernoulli source, which is a special case of the general periodic Markov source described in Section 2 and used as a source model in the simulation. As shown in Fig. 1, the $k$ th periodic Bernoulli source is governed by an underlying discrete-time $M^{(k)}$-state Markov chain with period $M^{(k)}$. If its underlying Markov chain is in the state $m^{(k)}\left(m^{(k)} \in\right.$ $\left.\left\{0, \ldots, M^{(k)}-1\right\}\right)$ in the $n$th slot, the source generates one cell with probability $\hat{\lambda}_{m^{(k)}}^{(k)}$ and it is in the state $m^{(k)} \oplus_{k} 1$ in the $(n+1)$ st slot with probability one. For the periodic Bernoulli sources, the PF eigenvalue and its associated left and right eigenvectors with the normalizing conditions are derived in [9].

In particular, the periodic Bernoulli sources which is used in the simulation are set as follows. Each source is described by the three parameters $l^{(k)}, p^{(k)}$ and $\gamma^{(k)}$. From state 1 through state $l^{(k)}$, it generates exactly one cell every $p^{(k)}$ slots with probability one. From state $l^{(k)}+1$ through state $M^{(k)}$, it generates one cell with probability $\gamma^{(k)}$. We consider a queue with a superposition of 7 identical periodic Bernoulli sources where we set $M^{(k)}=100, l^{(k)}=40$, $p^{(k)}=4$ and $\gamma^{(k)}=0.05$ for $k=1, \ldots, 7$. Throughout the simulation, the 
performance measure $J_{N_{0}, N}^{m}=\sum_{n=N_{0}}^{N_{0}+N-1} \operatorname{Pr}\left\{X_{n}>m\right\} / N$ where $m=20$, $N_{0}=4.0 \times 10^{3}$ and $N=1.0 \times 10^{6}$ will be estimated.

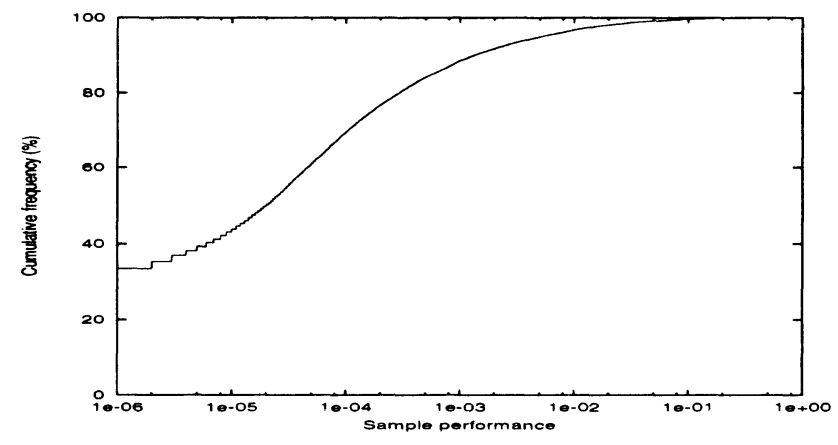

Figure 2 Cumulative frequency of the sample performance

Before examining the usefulness of the sampling technique, we will observe that the sample performance can drastically change depending on the combination of the initial states. Fig. 2 shows the cumulative frequency of the sample performance $\sum_{n=N_{0}}^{N_{0}+N-1} \mathbf{1}_{\left\{X_{n}>m\right\}} / N$ for randomly chosen 20000 initial states (with the initial distribution $\mu$ ). In Fig. 2, we observe that the samples widely spread. While $50 \%$ of the samples are less than $2.00 \times 10^{-5}, 0.01 \%$ of the samples are greater than $1.97 \times 10^{-1}$. We can see that as mentioned in Introduction, there exist some bad initial state combinations which lead to extreme degradation of the system performance, although they are rarely realized. This makes it hard to get estimates with low variance by standard MC simulation.

Table 1.1 Simulation results

\begin{tabular}{ccc}
\hline Method & standard MC & MC using sampling technique \\
\hline Estimation & $1.85 \times 10^{-3}$ & $1.91 \times 10^{-3}$ \\
Variance & $9.10 \times 10^{-7}$ & $2.10 \times 10^{-8}$ \\
\hline
\end{tabular}

We now investigate the usefulness of the sampling technique for reducing the variance of the estimates. For this purpose, we obtain 100 estimates and compute the sample variance of these estimates. Each estimate is the sample mean of 200 samples (i.e., $N_{s}=200$ ). Table 1.1 displays the simulation results obtained through the standard MC simulation and the MC simulation using the sampling technique. In Table 1.1, we observe that the utilization of the sampling technique can reduce the variance of the estimates.

For both the standard $\mathrm{MC}$ simulation and the $\mathrm{MC}$ simulation using the sampling technique, the average total CPU time required to obtain an estimate was $523 \mathrm{sec}$. Thus, the overhead computational cost to get a biased initial distribution is negligible in this case. We define the time-reliability product [3] as $($ time-reliability product $)=($ the average total CPU time required to obtain 
an estimate) $\times$ (the variance of the estimates). We then consider the speed-up factor [3], which is defined as the ratio of the time-reliability product of the standard MC simulation to that of the simulation using the sampling technique. In this case, the speed-up factor was 43.

\section{CONCLUSIONS}

For MC simulation of queues with a superposition of periodic and bursty sources, we have developed a sampling technique which replaces the initial distribution with a new biased one. The simulation result shows that the sampling technique is useful to reduce the variance of the estimates. We are now investigating the usefulness of the sampling technique combined with existing IS techniques which replace the state transition probabilities with biased ones.

\section{References}

[1] Abate, J., Choudhury, G. L., and Whitt W. (1994). "Asymptotics for steady-state tail probabilities in structured Markov chains of $M / G / 1$-type". Stoch. Mod., 10:99-144.

[2] Chang, C.-S. (1995). "Sample path large deviations and intree networks". Queueing Systems, 20:7-36.

[3] Devetsikiotis, M., and Townsend, J. K. (1993). "Statistical optimization of dynamic importance sampling parameters for efficient simulation of communication networks". IEEE/ACM Trans. Networking, 1:293-305.

[4] Duffield, N. G. (1994). "Exponential bounds for queues with Markovian arrivals" . Queueing Systems, 17:413-430.

[5] Falkenberg, E. (1994) "On the asymptotic behavior of the stationary distribution of Markov chains of $M / G / 1$-type". Stoch. Mod., 10:75-98.

[6] Glynn, P. W., and Iglehart, D. L. (1989). "Importance sampling for stochastic simulations". Management Sci., 35:1367-1392.

[7] Haraszti, Z., and Townsend, J. K. (1998). "The theory of direct probability redistribution and its application to rare event simulation". Proc. of ICC '98, 1443-1450.

[8] Ishizaki, F., and Takine, T. (1997). "Bounds for the tail distribution in a queue with the superposition of general periodic Markov sources". Proc. of INFOCOM '97, 1088-1095.

[9] Ishizaki, F., and Ohta, C. (1998). "Finding good combinations of initial phases for periodic sources in discrete-time queues". Proc. of ICC '98, 1510-1514.

[10] Ishizaki, F., (1999). "Sampling technique for queues with superposition of periodic and bursty sources". in preparation.

[11] Karlin, S., and Taylor, H. M. (1975). "A first course in stochastic processes, second edition". Academic press, San Diego.

[12] Neuts, M. F. (1989). "Structured stochastic matrices of M/G/l type and their applications". Marcel Dekker, New York.

[13] Rubinstein, R. Y. (1981). "Simulation and the Monte Carlo method". John Wiley \& Sons, New York.

[14] Sadowsky, J. S. (1991). "Large deviations theory and efficient simulation of excessive backlogs in a GI/GI/m queue". IEEE Trans. Autmat. Contr., 36:1383-1394.

[15] Villén-Altamirano, M., Martínez-Marrón, A., Gamo, J., Fernández-Cuesta, F. (1994). "Enhancement of the accelerated simulation method RESTART by considering multiple thresholds". Proc. of ITC 14, 797-810.

[16] Wang, Q., and Frost, V. S. (1993). "Efficient estimation of cell blocking probability for ATM systems". IEEE/ACM Trans. Networking, 1:230-235. 